\title{
Optimal Capacitor Placement for Voltage Stability Enhancement in Distribution Systems using BBO
}

\author{
S. Geethanjali \\ Assistant Professor \\ Department of Electrical Engineering \\ SRM University \\ Tamil Nadu, India
}

\author{
A. Sakthidasan \\ Teaching Fellow \\ Department of Electrical and Electronics \\ Engineering \\ UCEA (A Constituent College of Anna University, \\ Chennai), Arani, Tamil Nadu, \\ India
}

\begin{abstract}
The operating conditions of the present day distribution systems are closer to the voltage stability boundaries due to the ever increasing load demand. This article presents a biogeography based optimization (BBO) algorithm for determining the optimal locations and sizing of static and/or switched shunt capacitors with a view to enhance voltage stability of distribution systems. Biogeography deals with geographical distribution of biological species. Mathematical models of biogeography portray how species arises, migrates from one habitat to another and gets smeared out. The BBO algorithm searches for global solution through migration and mutation. The superiority of this approach is demonstrated by testing the algorithm on 15, 33 and 69-node distribution systems.
\end{abstract}

\section{General Terms}

Distribution systems

\section{Keywords}

voltage stability, radial distribution systems, capacitor placement

\section{NOMENCLATURE}

BBO biogeography based optimization

CC capacitor cost

CFC cost of the fixed capacitor $\times$ no. of fixed capacitors

CSC cost of the switched capacitor $\times$ no. of switched capacitors

$E^{\max } \quad$ maximum emigration rate

FIC fixed installation cost at each node

HSI habitat suitability index

$I^{\max } \quad$ maximum immigration rate

Iter $^{\max }$ maximum number of iterations

$K_{e} K_{p} K_{c} \& K_{v} \quad$ constants for energy loss cost, peak load loss cost, capacitor cost and VSC

$K_{q} \quad$ penalty factor for reactive support limit constraint

$m n c_{j} \quad$ maximum number of capacitors banks to be

connected at node- $j$

NCB number of capacitor banks

$n n \quad$ number of nodes in the system

$n h \quad$ number of habitats

$n \quad$ total number of species in the habitat

neh number of elite habitats

nll number of load levels

\footnotetext{
$N_{C i, j} \quad$ number of capacitor banks for $i$-th load level at $j$-th ranked node.

PCPA proposed capacitor placement algorithm

$P^{s}(t) \quad$ probability that the habitat contains exactly $\mathrm{S}$ species at time $t$

$P^{s} \quad$ species count probability

$P^{\text {mod }} \quad$ habitat modification probability

$P^{m} \quad$ mutation probability

$P_{i}^{L} \quad$ net power loss in the system at $i$-th load level

$P^{L} \quad$ peak power loss in the system

$P_{m}+j Q_{m} \quad$ real and reactive powers at the receiving end of branch- $m$

$P_{L-m}+j Q_{L-m} \quad$ real and reactive power load at node- $m$

$Q^{R} \quad$ available rating of a capacitor

$Q_{C i, j} \quad$ reactive power support required for $i$-th load level at node- $j$

$Q_{L j} \quad$ reactive power load at node- $j$

$r_{m}+j x_{m}$ resistance and reactance of branch- $m$ connected between nodes- $k$ and $m$

$S \quad$ species in the habitat

SIV suitability index variable

TCB type of capacitor banks

$T_{i} \quad$ duration of $i$-th load level

$t$ and $\Delta t$ time and change in time respectively

VM voltage magnitude

VS voltage stability

$V M^{L} \quad$ lowest value of VM in the system

VSC voltage stability cost

$V S I \quad$ VSI at node- $m$

$V S I^{L} \quad$ lowest value of VSI in the system

$\Psi \quad$ objective function to be minimized

$\lambda$ and $\mu$ immigration and emigration rates respectively

$\eta \quad$ a set of nodes considered for $\mathrm{CP}$
}

\section{INTRODUCTION}

The phenomenon of voltage instability in power systems is characterized by a monotonic voltage drop, which is slow at first, becomes abrupt after some time and is generally 
triggered by some form of disturbance or change in the operating conditions that create an increased demand for reactive power, which is in excess of what the system is capable of supplying. The other factors contributing to voltage collapse are generator reactive power/voltage control limits, load characteristics, characteristics of reactive compensation devices and the action of voltage control devices such as transformer under load tap changers [1-2]. In recent years, the distribution systems experience a sharp increase in load demand on account of the extensive growth of the utilities. Besides, with the advent of deregulation in the power industry, there is a greater focus on managing the network assets efficiently rather than reinforcing the network's capacity. The operating conditions are thus more and more closer to the voltage stability (VS) boundaries. In addition, distribution networks are subjected to distinct load changes everyday. In certain industrial areas, it is observed that under certain critical loading conditions, the distribution system suffers from voltage collapse [3]. Over the years, a number of approaches besides various indices for studying the VS are suggested in the literature [4-6].

Shunt capacitors supply reactive power and boost local voltages. Though several attempts have emerged to use capacitor banks in distribution systems for power factor correction, feeder voltage control [1-2], loss minimization [7], reliability enhancement [8] and profitability enhancement [9], hardly any work is found involving them with a view of enhancing VS of distribution systems. Recently algorithms for enhancing the VS of transmission system by optimal CP have been discussed in [10]. A relationship between VS and loss minimization that facilitates maximizing VS through loss minimization is outlined in [11]. An elegant $\mathrm{CP}$ algorithm to enhance VS of distribution systems through obtaining a condition for maximizing VS is suggested in [12]. An analytical CP algorithm to enhance VS of distribution system by treating the reactive line flow of the weakest line as the decision variable is outlined in [13]. A CP algorithm based on a VS index (VSI) for determining the optimal locations and sizing of static and/or switched shunt capacitors in order to enhance VS in addition to improving the VP is developed in [14]. A simple CP technique with a view to avoid voltage collapse through linearsing a VSI and reactive power flow of the weakest line is proposed for distribution systems in [15]. However, there is still a need for efficient and economically justified solution strategies for enhancing the VS of the present day stressed distribution systems

Recently, a Biogeography-Based Optimization (BBO) modeled on the theory of biogeography, which is the study of the geographical distribution of biological organisms, has been proposed for solving optimization problems by Simon [16]. Like other evolutionary algorithms, BBO is a population based stochastic optimization technique, sharing information between candidate solutions based on their fitness values with a view of obtaining the global best solution. Since its introduction, it has been applied to a variety of problems including sensor selection [16], power system optimization [17-18], ground water detection [19] and satellite image classification [20].

A new BBO algorithm that uses the VSI suggested in [5], for optimal placement of static and/or switched shunt capacitors in radial distribution system for VS enhancement is proposed in this article. This method improves the voltage profile and reduces system losses in addition to enhancing VS. The method is tested on 15-, 33- and 69-node radial distribution systems and the results presented.

\section{BBO}

BBO, suggested by Dan Simon in 2008, is a stochastic optimization technique for solving multimodal optimization problems. It is based on the concept of biogeography, which deals with the distribution of species that depend on different factors such as rain fall, diversity of vegetation, diversity of topographic features, land area, temperature, etc. A larger number of species are found in favorable areas compared with that of a less favorable area. A habitat is defined as an island that is geographically isolated from other areas. The areas that are well suited as residents for species are said to have high Habitat Suitability Index $(H S I)$. The variables that characterize habitability are called suitability index variables ( SIVs). The large number of species on high $H S I$ islands has many opportunities to emigrate into neighboring habitats with less number of species and share their characteristics with those habitats. For this reason, habitats with low HSI have a high species immigration rate. The immigration and emigration process helps the species in the area with low HSI to gain good features from the species in the area with high HSI and makes the weak elements into strong. Besides it allows to retain good features of species in the area with high $H S I$. The rate of immigration $(\lambda)$ and the emigration $(\mu)$ are the functions of the number of species in the habitat. Fig. 1 shows the immigration and emigration curves indicating the movement of species in a single habitat.

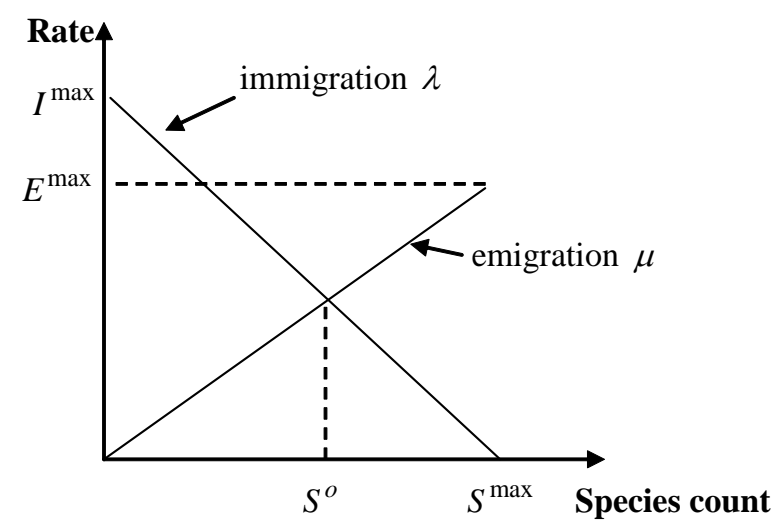

Fig.1 Species model of single habitat

In $\mathrm{BBO}$, a good solution is referred to an island with high $H S I$ and a poor solution to an island with low $H S I$. The poor solutions in islands with low HSI accept a lot of new features from good solutions in islands with high HSI and improve their quality. However, the shared features of the good solution still remain in the high HSI solutions. The concept of immigration and emigration is mathematically represented by a probabilistic model, which relates the probability $P^{s}(t)$ that a habitat contains exactly $S$ species at time $t$ with that of the probability $P^{s}(t+\Delta t)$ at time $(t+\Delta t)$, as

$$
\begin{aligned}
& P^{s}(t+\Delta t)=P^{s}(t)\left(1-\lambda_{s} \Delta t-\mu_{s} \Delta t\right)+ \\
& P^{s-1} \lambda_{s-1} \Delta t+P^{s+1} \mu_{s+1} \Delta t
\end{aligned}
$$

If time $\Delta t$ is so small that the probability of more than one immigration or emigration can be ignored then taking the limit of Eq. (1) as $\Delta t \rightarrow 0$ gives the following equation 


$$
\dot{P^{s}}=\left\{\begin{array}{lc}
-\left(\lambda_{s}+\mu_{s}\right) P^{s}+P^{s+1} \mu_{s+1} & S=0 \\
-\left(\lambda_{s}+\mu_{s}\right) P^{s}+P^{s+1} \mu_{s+1}+P_{s-1} \lambda_{s-1} & 1 \leq S \leq S^{\max } \\
-\left(\lambda_{s}+\mu_{s}\right) P^{s}+P^{s-1} \lambda_{s-1} & S=S^{\max }
\end{array}\right.
$$

The equation for emigration rate $\mu_{k}$ and immigration rate $\lambda_{k}$ for $k$-number of species is developed from Fig. 1 as

$$
\begin{gathered}
\mu_{k}=\frac{E^{\max }}{n} \\
\lambda_{k}=I^{\max }\left(1-\frac{k}{n}\right)
\end{gathered}
$$

When $E^{\max }=I^{\max }$, the immigration and emigration rates can be related as

$$
\lambda_{k}+\mu_{k}=E^{\max }
$$

The concept of BBO is based on the mechanisms of migration and mutation as discussed below.

\subsection{Migration}

A population of candidate solutions can be represented as vectors of real numbers in BBO algorithm. Each real number in the array is considered as an $S I V$, which is then used to evaluate the fitness of each candidate solution, denoted by $H S I$. High $H S I$ represents a better quality solution and low HSI denotes an inferior solution. The emigration and immigration rates of each solution are probabilistically used to control the sharing of features between habitats through a habitat modification probability, $P^{\mathrm{mod}}$. If a given solution $S_{i}$ is selected for modification, then its $\lambda$ is used to probabilistically decide whether or not to modify each $S I V$ in that solution. After selecting the $S I V$ for modification, $\mu$ of other solutions are used to select which of the solutions among the habitat set will migrate randomly chosen SIVS to the selected solution $S_{i}$. Some kind of elitism, which retains

the best habitat having highest $H S I$ without performing migration operation, is used in order to prevent the best solutions from being corrupted.

\subsection{Mutation}

The cataclysmic events that drastically change the HSI of a habitat is represented by mutation of $S I V$ and species count probabilities are used to determine mutation rates. The

probability of each species count, $P^{s}$ given by Eq. (2), indicates the likelihood that it exists as a solution for a given problem. If the probability of a given solution is very low, then that solution is likely to mutate to some other solution. Similarly if the probability of some solution is high, then that solution has very little chance to mutate. So, it can be said that very high $H S I$ solutions and very low $H S I$ solutions have less chance to create more improved $S I V$ in the later stage. But the medium HSI solutions have better chance to create much better solutions after mutation operation. Mutation rate of each set of solution can be calculated in terms of species count probability using the following equation:

$$
m(S)=m^{\max }\left(\frac{1-P^{s}}{P^{\max }}\right)
$$

This mutation scheme tends to increase diversity among the population, avoids the dominance of highly probable solutions and provides a chance of improving the low HSI solutions even more than they already have.

\section{PROPOSED ALGORITHM}

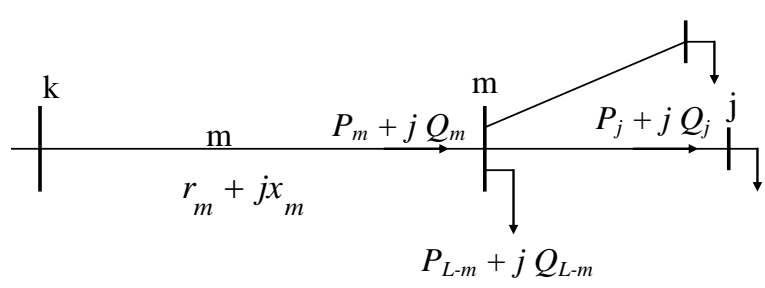

Fig. 2 Sample Distribution Line

The philosophy of Proposed Capacitor Placement Algorithm (PCPA) is to identify a set of weaker nodes from VS point of view and determine the locations, types, number and sizes of fixed and switchable capacitors to be installed through BBO with an objective to reducing the energy losses, decreasing the peak load loss, lowering the capacitor cost and improving the VS. It uses a VSI, suggested in [5], that varies between unity at no load and zero at voltage collapse point for determining the weaker nodes. The VSI at node- $m$ of Fig. 2 is determined by

$$
V S I_{m}=V_{k}^{4}-4\left\{P_{m} x_{m}-Q_{m} r_{m}\right\}^{2}-4\left\{P_{m} r_{m}+Q_{m} x_{m}\right\} V_{k}^{2}
$$

The node with lowest VSI value is identified as the weakest node. In addition nodes can be ranked based on the VSI values.

There are two components of losses in any distribution line. The first one is the losses associated with real current flow and the other associated with reactive current flow. It is known that the real current flow cannot be eliminated for a given loading pattern. But the reactive currents can be supplied at appropriate nodes and their flow through the distribution lines can be reduced, thereby minimizing the associated reactive power losses and enhancing VS. It is obvious that the losses due to reactive current flow can be completely eliminated by placing capacitor banks at all the nodes to supply local reactive power. However, it may not be practical to place capacitor banks all the nodes in the system.

\subsection{Representation BBO Variables}

The location for CP may be identified by computing the VSI, given by Eq. (7), and ranking the nodes by arranging the VSI values in ascending order. The node that is ranked first is the most sensitive node from VS point of view and requires priority in providing compensation. The first few nodes in the ranking list are selected for $\mathrm{CP}$. Let us assume $n c n$ - nodes are selected for compensation. It should be noted that the BBO algorithm determines the amount of VAR support required at each load level only at the selected nodes. The number of decision variables of the problem thus depends on the number of load levels considered over the planning period and the number of nodes considered for CP. The SIV of each habitat can therefore be represented as

Habitat $=$ 


\begin{tabular}{|c|c|c|c|c|c|}
\hline$N_{C 1,1}$ & $N_{C 1,2}$ & $\cdots$ & $N_{C 1, j}$ & $\cdots$ & $N_{C 1, n c n}$ \\
\hline$\cdots$ & $\cdots$ & $\cdots$ & $\cdots$ & $\cdots$ & $\cdots$ \\
\hline$N_{C i, 1}$ & $N_{C i, 2}$ & $\cdots$ & $N_{C i, j}$ & $\cdots$ & $N_{C i, n c n}$ \\
\hline$\cdots$ & $\cdots$ & $\cdots$ & $\cdots$ & $\cdots$ & $\cdots$ \\
\hline$N_{C \text { nll,1 }}$ & $N_{C \text { nll,2 }}$ & $\cdots$ & $N_{C \text { nll,j }}$ & $\cdots$ & $N_{C \text { nll,ncn }}$ \\
\hline
\end{tabular}

The capacitive support required for $i$-th load level at $j$-th ranked node can be calculated by

$$
Q_{C i, j}=N_{C i, j} \times Q^{R}
$$

At each node, there is always a maximum limit for providing the reactive power support for practical and economic reasons. Therefore, the maximum number of capacitors banks to be connected at node- $j$ is limited to $m n c_{j}$ and the limit constraints on the decision variables are

$$
0 \leq N_{C i, j} \leq m n c_{j} \quad i=1,2, \cdots, n l l
$$

\subsection{Problem Objectives and Constraints}

The objective of the proposed formulation is to minimize the energy losses, decrease peak load loss and lower the capacitor cost and improve the VS by providing appropriate amount of reactive power support at the selected nodes. It can be written as

$\operatorname{Min} \Psi=K_{e} \sum_{i=1}^{n l l} T_{i} P_{i}^{L}+K_{p} P_{o}^{L}+K_{c} C C+K_{v} V S C$

where

$$
V S C=\frac{1}{\prod_{m=1}^{n n} V S I_{m}}
$$$$
C C=\mathrm{FIC}+\mathrm{CFC}+\mathrm{CSC}
$$

At any load level, the net reactive power support should not exceed the total reactive power load in the system. The reactive power limit constraint can thus be modeled as

$$
\sum_{j \in \eta} Q_{C i, j}=\sum_{j=2}^{n n} Q_{L j} \quad i=1,2, \cdots, n l l
$$

\subsection{Habitat Suitability Index}

The BBO searches for optimal solution by maximizing a $H S I$ function, which is formulated from the objective function of Eq. (11) and reactive power limit constraint of Eq. (13) as

$$
\text { Max } H S I=\frac{1}{1+\Phi}
$$

Where

$$
\operatorname{Min} \Phi=K_{e} \sum_{i=1}^{n l l} T_{i} P_{i}+K_{p} P_{o}+K_{c} C C+K_{v} V S C+
$$

$$
K_{q}\left\{\sum_{j \in \eta} Q_{C i, j}-\sum_{j=2}^{n n} Q_{L j}\right\}^{2}
$$

\subsection{Repair Algorithm}

The number of capacitor banks required at any node for lower load level should be less than that of higher load levels and vice versa. Let the first row of each habitat denotes the lowest load level, the second row next higher load level and so on for convenience. The following repair mechanism is performed for each habitat.

for $j=1: n c n$

$$
\begin{aligned}
& \text { for } k=n l l:-1: 2 \\
& \qquad \begin{array}{r}
\text { set } M=N_{C k, j} \\
\text { if } N_{C k-1, j} \geq M, \\
\text { replace } N_{C k-1, j} \text { by a random } \\
\text { number in the range }(0, M)
\end{array}
\end{aligned}
$$

end

end

end

\subsection{Stopping Criteria}

The process of generating new habitats can be terminated either after a fixed number of iterations or if there is no further significant improvement in the global best solution.

\subsection{Algorithm}

1. Read the line data and load data at different load levels.

2. Choose maximum number of capacitors banks, $m n c_{i}$ to be connected at each node- $i$.

3. Choose heuristically the number of nodes, $n c n$, for VAR support

4. Carryout distribution power flow [23] and compute VSI using Eq. (7)

5. Rank the VSI values in ascending order and choose nodes corresponding to the first $n c n$-values in the ranked list.

6. Set the iteration counter iter $=1$

7. Choose the BBO parameters such as $n h$, neh, $I^{\max }$, $E^{\max }, P^{\bmod }, P^{m}$ and Iter $^{\max }$.

8. Randomly generate integer values to denote $S I V S$ of each habitat in the range of $\left[0, m n c_{i}\right]$ so as to form habitat matrix containing $n h$-habitats.

9. Repair the habitats using the procedure described in section 3.3

10. Perform the following for each habitat 
- For each load level,

- Add the reactive power support equal to $\left\{N_{C, k} \times Q_{C}\right\}$ at the respective node for $k=1,2, \cdots, n c n$

- Carryout Distribution Power Flow

- $\quad$ Evaluate VSI using Eq. (7)

- Evaluate $H S I$ through Eq. (14)

11. Identify neh habitats having highest $H S I$ and retain them as it is without making any modifications

12. Evaluate $\mu$ and $\lambda$ for each habitat.

13. Perform migration probabilistically on those SIVs of non-elite habitats based on $P^{\bmod }$.

14. Perform mutation probabilistically on those SIVs of non-elite habitats based on $P^{m}$.

15. Check for convergence. i.e., check whether any one of the following criteria is satisfied.

- the number of iterations since the last change of the best solution is greater than a pre-specified number or

- the number of iterations reaches Iter $^{\max }$.

16. If converged, the solution corresponding to the elite habitat is the optimal solution; else, increment iteration counter $\{$ iter $=$ iter +1$\}$ and go to step (9)

\section{RESULTS AND DISCUSSIONS}

The PCPA is tested on 15-, 33- and 69-node distribution systems. The line and load data for these three systems are obtained from the references [21] and [22]. The size of the capacitor bank considered in this study is $150 \mathrm{kVAR}$ and its multiples. The cost of fixed and switched capacitors is considered as $\$ 900$ and $\$ 1200$ respectively. The results are obtained for light, medium, full and over load conditions by multiplying the base-load by a factor $0.5,0.8,1.0$ and 1.1 respectively. The duration of each interval is taken as 1000 , 3000,3760 and 1000 hours. It uses the distribution power flow suggested in [23].

15 node test system: The algorithm is run several times by changing values from 5 to 10 and the results obtained. It is observed that the losses are reduced to reasonably low level when equals ten. The net reactive power load in the system and minimum VAR support required at each selected nodes for different loading conditions are given in Table 1. It is to be noted that the net VAR support at each loading level is less than the reactive power load in the system. The capacitor banks to provide VAR support at low load level is chosen as fixed type and the rest as switched type. The classification of fixed and switch type are given in Table 2 .

$33 \& 69$ node test systems: The net reactive power load in the system and minimum VAR support required at each selected nodes for different loading conditions when equals 15 and 14 respectively for 33 and 69-node systems respectively are given in Tables 3 and 6 . The classification of fixed and switch type are given for 33 and 69 node systems are given in Tables 4 and 5 respectively.

The losses, the lowest VM and the lowest VSI for all the systems under study before and after $\mathrm{CP}$ are graphically displayed in Figs. 3, 4 and 5 respectively. It is clear from these figures that the proposed method minimizes the losses, improves the voltage profile and enhances the VS.

Table 1 Requirement of VAR compensation for 15-node system

\begin{tabular}{|c|c|c|c|c|c|c|c|c|c|c|c|c|c|}
\hline \multicolumn{2}{|c|}{ Node No } & $\mathbf{9}$ & $\mathbf{8}$ & $\mathbf{5}$ & $\mathbf{6}$ & $\mathbf{4}$ & $\mathbf{7}$ & $\mathbf{3}$ & $\mathbf{1 3}$ & $\mathbf{2}$ & $\mathbf{1 4}$ & Net Reactive Power load & Net VAR support \\
\hline \multirow{4}{*}{ NCB } & Light load & 4 & 5 & 1 & 5 & 5 & 2 & 3 & 7 & 4 & 5 & 6.26 & 6.15 \\
\cline { 2 - 14 } & Medium load & 7 & 7 & 2 & 8 & 8 & 4 & 4 & 10 & 7 & 9 & 10.01 & 9.90 \\
\cline { 2 - 13 } & Full load & 8 & 9 & 3 & 10 & 9 & 5 & 9 & 10 & 10 & 10 & 12.51 & 12.45 \\
\cline { 2 - 12 } & Over load & 9 & 10 & 5 & 10 & 10 & 5 & 10 & 10 & 10 & 10 & 13.76 & 13.35 \\
\hline
\end{tabular}

Table 2 Type and Size of Capacitor placed for 15 node system

\begin{tabular}{|c|c|c|c|c|c|c|c|c|c|c|c|}
\hline \multicolumn{2}{|c|}{ Node No } & $\mathbf{9}$ & $\mathbf{8}$ & $\mathbf{5}$ & $\mathbf{6}$ & $\mathbf{4}$ & $\mathbf{7}$ & $\mathbf{3}$ & $\mathbf{1 3}$ & $\mathbf{2}$ & $\mathbf{1 4}$ \\
\hline \multirow{2}{*}{ TCB } & Fixed & 4 & 5 & 1 & 5 & 5 & 2 & 3 & 7 & 4 & 5 \\
\cline { 2 - 10 } & Switched & 5 & 5 & 4 & 5 & 5 & 3 & 7 & 3 & 6 & 5 \\
\hline
\end{tabular}

Table 3 Requirement of VAR compensation for 33-node system

\begin{tabular}{|c|c|c|c|c|c|c|c|c|c|c|c|c|c|c|c|c|c|c|}
\hline \multicolumn{2}{|c|}{ Node No } & 17 & 16 & 15 & 14 & 13 & 12 & 32 & 31 & 30 & 11 & 10 & 9 & 29 & 28 & 8 & $\begin{array}{c}\text { Reactive } \\
\text { Power load }\end{array}$ & $\begin{array}{c}\text { Net VAR } \\
\text { support }\end{array}$ \\
\hline \multirow{4}{*}{$\mathrm{NCB}$} & Light load & 2 & 0 & 1 & 2 & 2 & 1 & 5 & 6 & 9 & 1 & 1 & 1 & 9 & 7 & 7 & 11.50 & 8.10 \\
\hline & $\begin{array}{l}\text { Medium } \\
\text { load }\end{array}$ & 4 & 0 & 1 & 3 & 2 & 2 & 10 & 9 & 9 & 1 & 3 & 8 & 10 & 9 & 10 & 18.40 & 12.15 \\
\hline & Full load & 4 & 0 & 1 & 3 & 2 & 2 & 10 & 9 & 9 & 1 & 6 & 10 & 10 & 9 & 10 & 23.00 & 12.90 \\
\hline & Over load & 4 & 2 & 1 & 6 & 2 & 3 & 10 & 10 & 10 & 3 & 9 & 10 & 10 & 10 & 10 & 25.30 & 15.00 \\
\hline
\end{tabular}

Table 4 Type and Size of Capacitor placed for 33 node system

\begin{tabular}{|c|c|c|c|c|c|c|c|c|c|c|c|c|c|c|c|c|}
\hline \multicolumn{2}{|c|}{ Node No } & 17 & 16 & 15 & 14 & 13 & 12 & 32 & 31 & 30 & 11 & 10 & 9 & 29 & 28 & 8 \\
\hline \multirow{2}{*}{ TCB } & Fixed & 2 & 0 & 1 & 2 & 2 & 1 & 5 & 6 & 9 & 1 & 1 & 1 & 9 & 7 & 7 \\
\hline & Switched & 2 & 2 & 0 & 4 & 0 & 2 & 5 & 4 & 1 & 2 & 8 & 9 & 1 & 3 & 3 \\
\hline
\end{tabular}


Table 5 Requirement of VAR compensation for 69-node system

\begin{tabular}{|c|c|c|c|c|c|c|c|c|c|c|c|c|c|c|c|c|c|}
\hline \multicolumn{2}{|c|}{ Node No } & 64 & 63 & 62 & 61 & 60 & 59 & 58 & 57 & 56 & 26 & 25 & 24 & 23 & 22 & $\begin{array}{c}\text { Reactive } \\
\text { Power load }\end{array}$ & $\begin{array}{c}\text { Net VAR } \\
\text { support }\end{array}$ \\
\hline \multirow{4}{*}{ NCB } & Light load & 6 & 7 & 10 & 10 & 5 & 2 & 0 & 1 & 7 & 0 & 0 & 1 & 2 & 7 & 13.47 & 8.70 \\
\hline & Medium load & 10 & 10 & 10 & 10 & 10 & 10 & 5 & 3 & 10 & 1 & 0 & 1 & 4 & 10 & 21.56 & 14.10 \\
\hline & Full load & 10 & 10 & 10 & 10 & 10 & 10 & 10 & 10 & 10 & 1 & 0 & 3 & 8 & 10 & 26.95 & 16.80 \\
\hline & Over load & 10 & 10 & 10 & 10 & 10 & 10 & 10 & 10 & 10 & 1 & 0 & 3 & 8 & 10 & 29.64 & 17.25 \\
\hline
\end{tabular}

Table 6 Type and Size of Capacitor placed for 69 node system

\begin{tabular}{|c|c|c|c|c|c|c|c|c|c|c|c|c|c|c|c|}
\hline \multicolumn{2}{|c|}{ Node No } & $\mathbf{6 4}$ & $\mathbf{6 3}$ & $\mathbf{6 2}$ & $\mathbf{6 1}$ & $\mathbf{6 0}$ & $\mathbf{5 9}$ & $\mathbf{5 8}$ & $\mathbf{5 7}$ & $\mathbf{5 6}$ & $\mathbf{2 6}$ & $\mathbf{2 5}$ & $\mathbf{2 4}$ & $\mathbf{2 3}$ & $\mathbf{2 2}$ \\
\hline \multirow{2}{*}{ TCB } & Fixed & 6 & 7 & 10 & 10 & 5 & 2 & 0 & 1 & 7 & 0 & 0 & 1 & 2 & 7 \\
\cline { 2 - 33 } & Switched & 4 & 3 & 0 & 0 & 5 & 8 & 10 & 9 & 3 & 1 & 0 & 2 & 6 & 3 \\
\hline
\end{tabular}

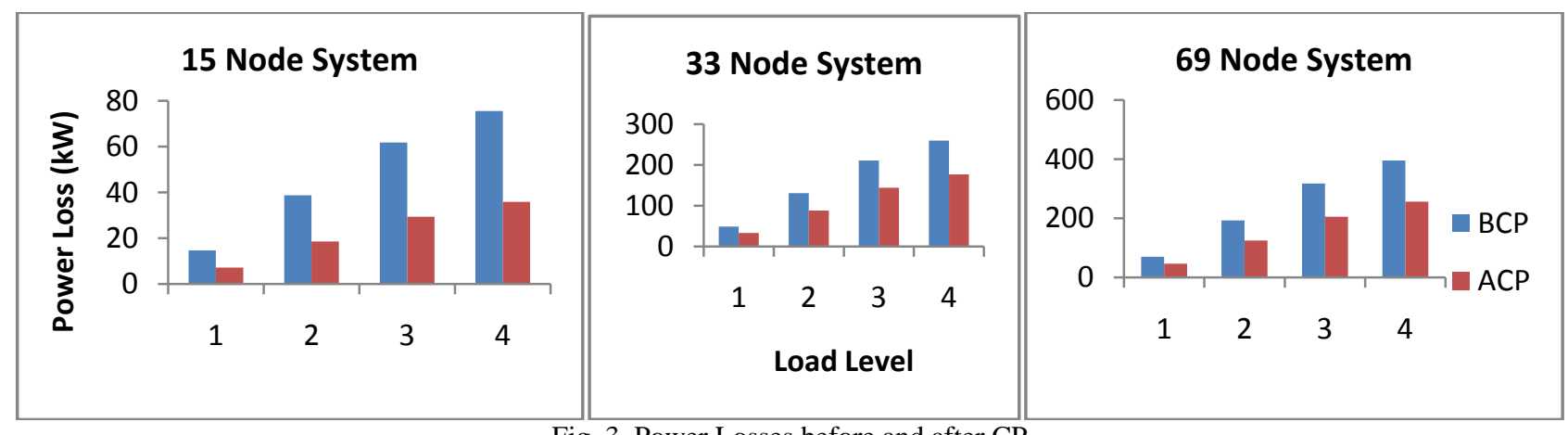

Fig. 3 Power Losses before and after CP
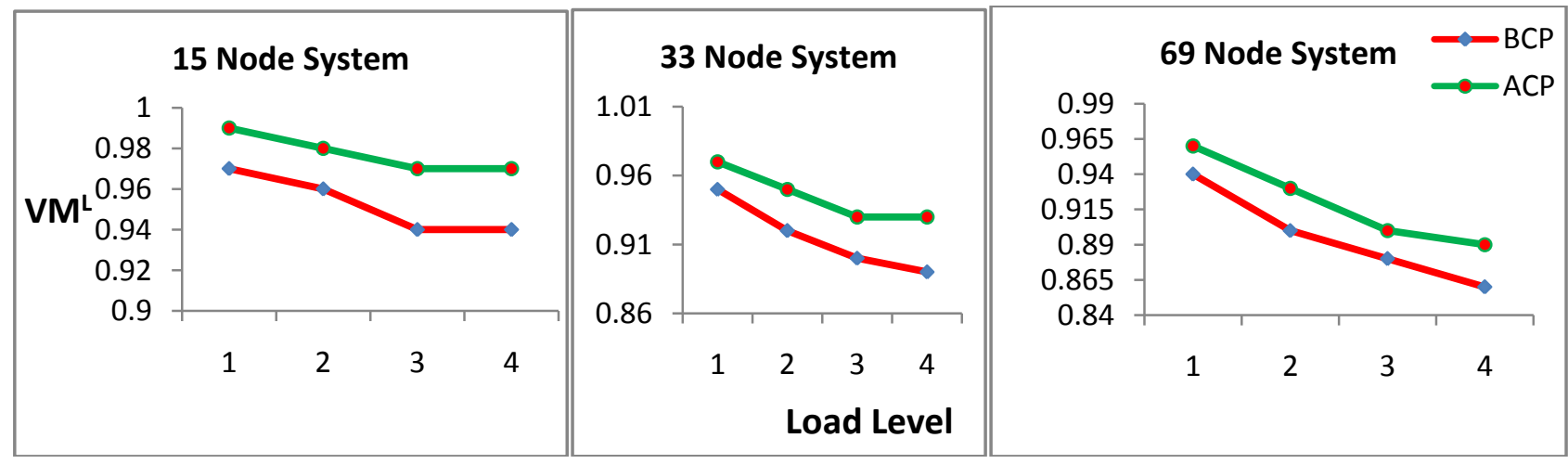

Fig. 4 Lowest VM in the system before and after CP

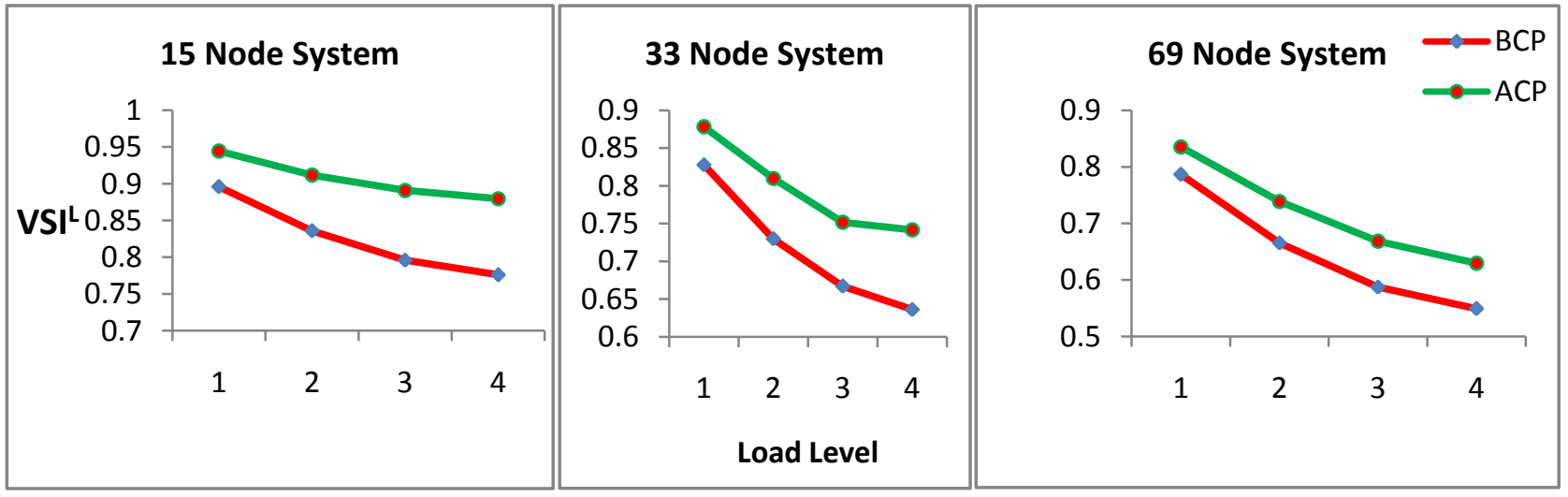

Fig. 5 Lowest VSI in the system before and after CP

\section{CONCLUSIONS}

A CP algorithm using BBO for radial distribution system has been developed. This method has been framed with a view to find the optimal locations and determine the size and type of capacitor banks to be placed in order to minimize losses besides enhancing the VS and improving the voltage profile.
The algorithm has been coined to place capacitors at a set of weaker nodes. The algorithm will be suitable for practical implementation on systems of any size on account of the ease of the computational procedure and the flexibility with which it can be incorporated. The developed method can be extended to place static var compensators (SVC) for better 
performances besides adaptively adjusting the BBO parameters.

\section{ACKNOWLEDGMENTS}

The authors gratefully acknowledge the authorities of SRM University, Chennai and Constituent College of Anna University, Arani, Tamil Nadu for their continued support, encouragement and the facilities provided to carry out this work..

\section{REFERENCES}

[1] Kundur. P, Power system stability and control, McGrawHill, Inc., 1993.

[2] C.W. Taylor, Power System Voltage Stability, McGrawHill, Inc., 1994.

[3] R.B. Prada and L.J. Souza, Voltage stability and thermal limit: constraints on the maximum loading of electrical energy distribution feeders, IEE Proc. Gen. Trans and Dist., Vol. 45 (5), pp. 573-77, 1998.

[4] A.K. Sinha and D. Hazarika, A comparative study of voltage stability indices in a power system, Electrical Power and Energy Systems, Vol. 22(1), pp. 589-596, 2000.

[5] M. Chakravorty and D. Das, "Voltage stability analysis of radial distribution networks," International Journal of Electrical Power and Energy Systems, Vol. 23(2), pp. 129-135, 2001.

[6] Wang. Y, Wenyuan Li, Jiping Lu, A new node voltage stability index based on local voltage phasors, Electric Power System Research, Vol. 79(1), pp. 265-271, 2009.

[7] S.K. Bhattacharya and S.K. Goswami, A new fuzzy based solution of the capacitor placement problem in radial distribution system, Expert Systems with Applications, Vol. 36(3), pp. 4207-12, 2009.

[8] A.H. Etemadi and M. Fotuhi-Firuzabad, Distribution system reliability enhancement using optimal capacitor placement, IEE Proc. Gener. Transm. Distrib., Vol. 2(5), pp. 621-631, 2008.

[9] J.C. Hernandez, A. Medina and F. Jurado, Optimal allocation and sizing for profitability and voltage enhancement of PV systems on feeders, Renewable Energy Journal, Vol. 32(10), pp. 1768-1789, 2007.

[10] P. K. Satpathy, D. Das and P. B. Dutta Gupta, Critical switching of capacitors to prevent voltage collapse, Electric Power Systems Research, Vol. 71(1), pp. 11-20, 2004.

[11] M. A. Kashem and M. Moghaavvemi, Maximising radial voltage stability and load balancing via loss minimisation in distribution networks, Proceedings of Energy Management and Power Delivery, Vol. 1, pp. 91-96, March 1998
[12] G. Mohan and P. Aravindhababu, "A novel capacitor placement algorithm for voltage stability enhancement in distribution systems", International Journal of Electronics Engineering, Vol.1(1), pp. 83-87, 2009.

[13] G. Mohan and P. Aravindhababu, "Optimal capacitor placement for voltage stability enhancement in distribution systems", Journal of Engineering and Applied Sciences, Vol. 4(2), April 2009.

[14] M. Arun and P. Aravindhababu. (2010). An elegant VAR support algorithm for voltage stability enhancement of distribution systems, Journal of Electrical Engineering, ISSN 1582-4594, 10(1), 59-64.

[15] G. Mohan and P. Aravindhababu, "Optimal Locations and Sizing of Capacitors for Voltage Stability Enhancement in Distribution Systems", International Journal of Computer Applications, Vol. 1(4), pp.55-67, 2010.

[16] D. Simon. (2008) Biogeography-based optimization, IEEE Transactions on Evolutionary Computation, 12(6), 702-713.

[17] Rarick. R, Simon. D, Villaseca. F, and Vyakaranam. B. (2009) Biogeography-based optimization and the solution of the power flow problem, IEEE Conference on Systems, Man, and Cybernetics, San Antonio, TX, 10291034.

[18] S.Rajasomashekar and P. Aravindhababu. (2012). Biogeography-based optimization technique for best compromise solution of economic emission dispatch, Swarm and Evolutionary Computations, 7: 47-57.

[19] Kundra. H, Kaur. A and Panchal. V. (2009) An integrated approach to biogeography based optimization with case based reasoning for retrieving groundwater possibility, in: 8th Annual Asian Conference and Exhibition on Geospatial Information, Technology and Applications.

[20] Panchal. V, Singh. P, Kaur. N and Kundra. H. (2009) Biogeography based satellite image classification, International Journal of Computer Science and Information Security, 6(2), 269-274.

[21] B. Venkatesh and R. Ranjan, "Optimal radial distribution system reconfiguration using fuzzy adaptation of evolutionary programming", Journal of Electrical Power and Energy Systems, Vol. 25 (10), pp. 775-780, 2003.

[22] S. Sivanagaraju, N. Visali, V. Sankar and T. Ramana, “ Enhancing voltage stability of radial distribution systems by network reconfiguration", Electric power components and systems, Vol. 33(5), pp. 539-50, 2004.

[23] P. Aravindhababu and R. Ashokkumar, A fast decoupled power flow for distribution systems, Electric Power Components and Systems, Vol. 36(9), pp. 932-940, 2008. 